\title{
Human Musculoskeletal and Energetic Adaptations to Unilateral Robotic Knee Gait Assistance
}

\author{
Tomislav Bacek, Marta Moltedo, Ben Serrien, Kevin Langlois, Bram Vanderborght, Senior Member, IEEE, \\ Dirk Lefeber, Member, IEEE and Carlos Rodriguez-Guerrero
}

\begin{abstract}
Objective: This paper aims to analyse the human musculoskeletal and energetic adaptation mechanisms when physically interacting with a unilateral knee orthosis during treadmill walking. Methods: Test subjects participated in two walking trials, whereby the orthosis was controlled to deliver five predefined torque profiles of different duration (as \% of a gait cycle). The adaptations to assistive torques of different duration were analysed in terms of gait parameters, metabolic effort, and muscle activity. Results: Orthotic assistance's kinematic effects remain local to the assisted leg and joint, unlike the muscles spanning the knee joint, which engage in a balancing-out action to retain stability. Duration of assistive torque significantly affects only the timing of the knee joint's peak flexion angle in the stance phase, while the observed joint kinematics and muscle activity demonstrate different recovery times upon changing robotic support (washout effects). Conclusion: Human body adaptations to external robotic knee joint assistance during walking take place on multiple levels and to a different extent in a joint effort to keep the gait stable. Significance: This paper provides important insights into the human body's multiple adaptation mechanisms in the presence of external robotic assistance.
\end{abstract}

Index Terms-Assistive duration, Joint kinematics, Knee orthosis, Metabolic cost, Muscle activity.

\section{INTRODUCTION}

W ALKING is the most exercised daily activity. Over time, walking converged to a certain intra- and interlimb coordination level and, despite inherent variability, well-established patterns [1]. However, these patterns and coordination are modified when human motion is affected by, e.g., an interacting robot [2], [3], or neurological disorder [4]. Therefore, understanding different adaptation mechanisms humans use in such scenarios is crucial to minimise negative effects. In this paper, we focus on physical human-robot interaction (pHRI).

Walking adaptations happen on multiple levels and seem to follow a hierarchy [5]. A widely accepted approach to study these adaptations is to measure three aspects of walking: gait parameters (joint kinematics and spatio-temporal gait characteristics), muscle activity, and metabolic cost (MC) [6]. Given the intrinsic, albeit complex relationship between the three metrics, it is of high interest to investigate how they are affected (i) simultaneously and (ii) depending on the point of interaction with a wearable robot (WR). The reason for

T. Bacek and M. Moltedo were with the Department of Mechanical Engineering, Vrije Universiteit Brussel, and Flanders Make, Belgium. T. Bacek is now with the Department of Mechanical Engineering, University of Melbourne, Australia. Corresponding e-mail: tomislav.bacek@unimelb.edu.au

B. Serrien, K. Langlois, B. Vanderborght, D. Lefeber and C. RodriguezGuerrero are with Vrije Universiteit Brussel and Flanders Make. this is a dependency of human musculoskeletal and energetic adaptations during pHRI on robotic parameters (e.g., actuation profile timing, assistance magnitude, and actuation system) [7], study design [8], and assisted joint(s) [9].

Studies investigating simultaneous changes in all three metrics in general, and during pHRI at the knee level in particular, are missing in the literature. The adaptations in healthy persons during pHRI while walking have been studied in the case of the ankle [7], [10]-[15], hip [16], [17], and the hip-ankle multi-joint configuration [18]-[20], but are missing in the case of a knee joint. Those studies that do focus on the knee joint do so by studying either one [21]-[25] or two metrics [26]-[30], but never all three. This leaves gaps in our understanding of how humans adapt as a whole when interacting with an active knee orthosis, a scenario found in different applications and environments [24], [28], [31].

For example, Shirota [2] showed that kinematic effects of unilaterally wearing passive knee orthosis remain local to the interaction leg but provided no insights into the accompanying muscle activity changes. In studying the benefits of bilaterally assisting the knee during loaded uphill walking, MacLean [24] showed positive effects on the MC but provided no analysis on how such interaction affected gait patterns. Other research has shown that human kinematic and kinetic patterns remain invariant to parallel stiffness applied bilaterally at the knee joint level [25] but fell short of putting this into the perspective of muscle activity. And while undeniably invaluable, the results of such studies provide an incomplete picture of the implications of pHRI at the knee level. Superimposing these results, albeit tempting, should be taken with caution due to multiple factors differing across studies that inevitably affect study outcomes.

In that context, this work's main contribution is the analysis of the human musculoskeletal and energetic adaptations while unilaterally assisting the knee during treadmill walking. The analysis presents the effects of such pHRI on all three discussed metrics while answering two unanswered yet relevant questions. On the one hand, it investigates whether the locality of kinematic effects holds during unilateral interaction with an active knee orthosis, extending the work of Shirota [2]. On the other hand, it examines the implications of changing the assistive torque duration (as \% of the gait cycle), which plays a major role in assisting the hip and ankle joints [7]. To that end, we hypothesised that (i) unilateral knee assistance would only affect kinematics and muscle activity of the assisted leg, and (ii) the human body would show different musculoskeletal and energetic adaptations when changing assistive torque duration. 


\section{Methods}

\section{A. Knee joint orthosis}

The knee joint orthosis used to assist the participants' left leg (hereafter: assisted leg) consists of an actuator, interface (cuffs and frame), and connections between the two (Fig. 1). The actuator's defining features are a simple rotational joint (viable choice for knee orthosis [25], [32]), anthropomorphic shape designed to minimise potential negative effects of its $2.2 \mathrm{~kg}$ mass [33], and a spring in series with an EC motor to deliver torque in the sagittal plane (see [34], [35] for detailed actuator specifications and performance).

The interface is custom-designed using 3D leg scans [36] and shaped to accommodate muscle activity electrodes. To comfortably fit people of different leg sizes, a cuff basemodel was scaled and fitted with soft padding. In addition, the actuator-interface fixation points (a keyed coupling with a screw) allow relative motion between the actuator and cuffs. Finally, the two shank cuffs are connected using a steel plate (Fig. 1.a, 1.b) heat-treated to be stiff in the sagittal plane while allowing some flexibility in the other two biological planes.

\section{B. Control architecture}

The control architecture consists of a low, middle, and high-level layer. The high-level layer sets desired torque and sends it as an input to the mid-level controller, which uses an experimentally-based actuator model to convert this into the desired motor position. A simple proportional gain is then used to map this position into the desired motor velocity. The motor is controlled at a low level using a controller provided by the manufacturer. This cascaded controller allows reliable delivery of any desired torque within the actuator's hardware/software limitations without measuring the torque [35].

The high-level controller assists the knee during the early stance gait phase when step-to-step transitions occur [37]. The controller is based on a finite state machine (FSM) with three states (Fig. 2): stance phase $S T$, swing phase $S W$, and pre-stance (i.e., terminal swing) phase pre-ST. The former two make the core of the controller. The latter was added to avoid assistance initiation delays at the start of a new gait cycle (triggered by the heel strike), which come from inherent hardware/software delays and person-specific stance flexion patterns [38]. Adding pre-ST phase circumvents delays by anticipating the heel strike's timing and starting the assistance build-up at $95 \%$ of the current cycle's expected duration. This ensures the actual assistance is felt by the user immediately after the heel strike. The heel strike (i.e., next step) is anticipated using (i) average step time calculated in real time and (ii) knee joint angle measured by the actuator (angle threshold value at $95 \%$ of a gait cycle duration taken from the literature [1]).

The FSM switches between states using sensor inputs from heel/toe footswitches and joint (actuator) position angle. At the start of each trial, participants walk at their preferred speed for one minute in a zero-torque mode. A moving 10-step window is used to calculate the average step duration, with steps being detected using the footswitches. At minute 1 , the controller initiates the assistance and monitors all available triggers (Fig.
2). Depending on the triggers' signal, the controller switches between the assistive mode during the pre-ST/ST phase and a zero-torque mode throughout the remainder of a gait cycle ( $S W$ phase). A change in the assistive torque duration between two consecutive blocks strictly happens during the $S W$ phase to avoid causing assistive impacts.

\section{Testing protocol}

The goal of the experimental protocol (Fig. 3.a) was to investigate human musculoskeletal and energetic adaptations in the presence of external knee assistance. In this work, the only assistive parameter changing is the torque duration (as \% of a gait cycle) of a bell-like torque trajectory (Fig. 3.b). This allows simplifying the analysis by isolating assistive duration effects from, e.g., assistive torque shape or amplitude.

The testing protocol comprises one session consisting of Subject Preparation and Gait Analysis parts (Table I). Both parts took place on the same day. The session always starts with the Subject Preparation part, which serves as a preparation for the actual measurements. During this part, the participants are equipped with surface EMG electrodes (bipolar, ConMed Corp., NY, USA), a gas measuring system (MetaMax 3B, Cortex, Germany), and retroreflective markers (spherical, $14 \mathrm{~mm}$ diameter, B\&L Engineering, CA, USA), and their baseline respiratory consumption is measured in standing. This part takes about 45 minutes.

Gait Analysis is a data collection part (Fig. 3.a), during which multiple levels of software-controlled assistive torque duration are tested. To do so in a short time, the Gait Analysis part is designed in a steady-state cost mapping (SSCM) fashion [42], a standard in the field [13]. Five assistive torque duration levels were swept through in a discrete-step manner, with each condition being applied during a 4-minute block (similar to [43]) - three minutes to reach metabolic steady-state [44] and one minute for data averaging. These blocks are divided between two assistive trials with a 10-minute break in between, taking about 60 minutes in total. Both trials start and end with a zero-torque (transparent) mode to allow the controller to set in and examine learning effects and fatigue (in terms of joint kinematics and muscle activity). The two trials' order is the same, while the assistive torque duration levels across the two trials are randomized for all participants. The assistance always starts at the moment of a heel-strike and ends between $18 \%$ (A1) and 34\% (A5) of a gait cycle (4\% intervals, Fig. 3.b). The peak torque amplitude is chosen as a fixed percentage of a person's weight, thus changing between subjects. In particular, the $0.05 \mathrm{Nm} / \mathrm{kg}$ peak was chosen, as this was the highest amplitude considered safe and comfortable by participants. The amplitude was limited by the knee hyperextension coming from impulse-like assistance during the shortest (A1) duration.

Walking trials took place on a dual-belt instrumented treadmill (X-Mill, Motek, Netherlands), which measured ground reaction forces (GRFs) subsequently used in gait segmentation. The participants walked at their preferred speed, coming at $3.6 \pm 0.3 \mathrm{~km} / \mathrm{h}(1 \pm 0.08 \mathrm{~m} / \mathrm{s})$ across all. The preferred speed is an average of two speeds - one self-selected while treadmill speed was gradually increasing $(0 \rightarrow 5 \mathrm{~km} / \mathrm{h})$, 

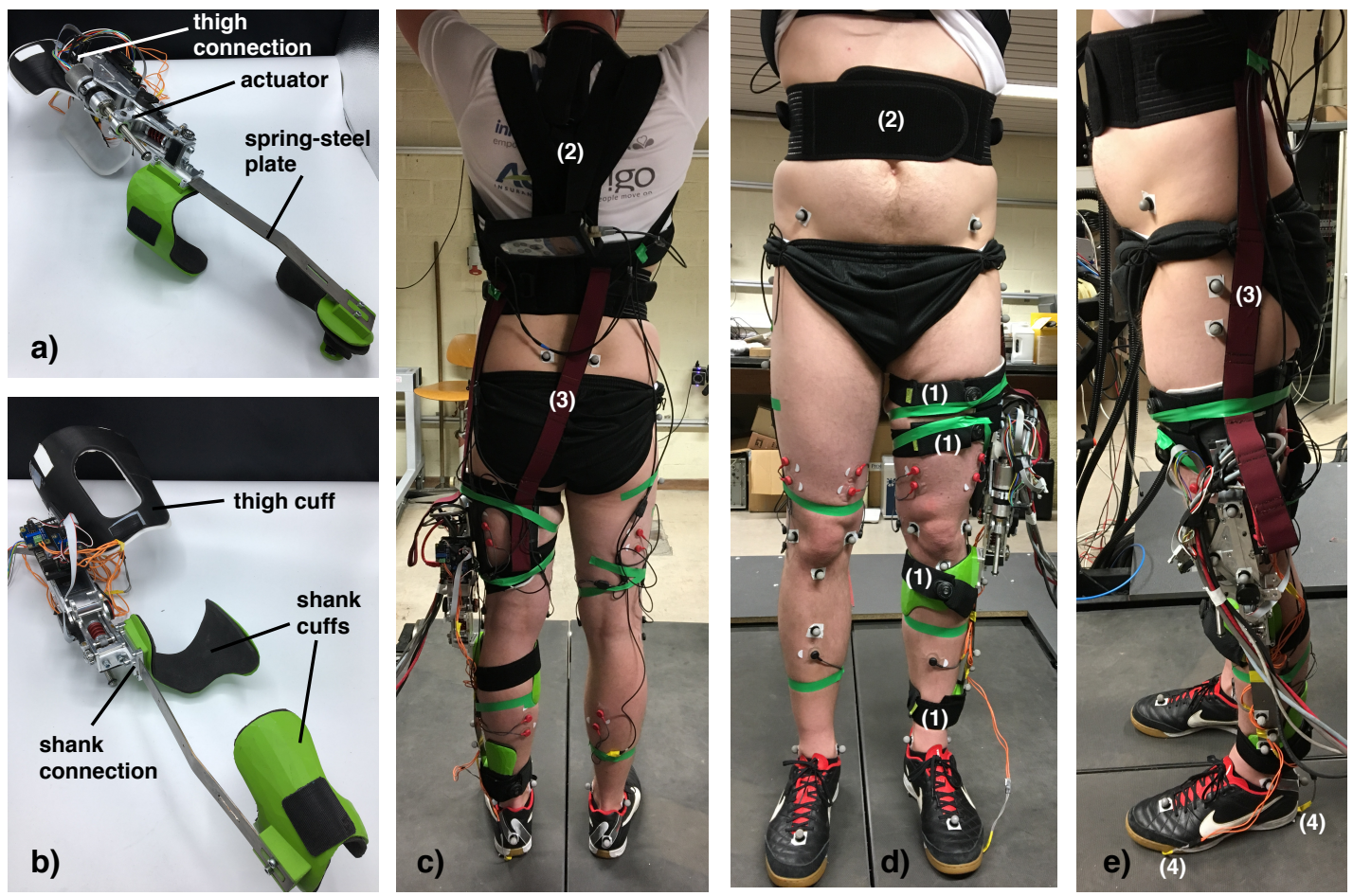

Fig. 1. Unilateral knee joint orthosis. $a, b$ ) The interface consists of the thigh cuff (black), two shank cuffs (green), and a steel frame. The actuator's position relative to the cuffs can be changed to fit people of different heights. The rotational joint design allows $\left[-5^{\circ}, 90^{\circ}\right]$ range of motion. $c$-e) The orthosis fitted to one of the participants. The cuffs were tightened to the leg using BOA straps (1) and attached to a commercially available posture corrector brace (2) using elastic bands (3) to prevent slippage. Each participant was equipped with electromyogram (EMG) electrodes (red and black) and Vicon retroreflective markers (grey) placed on their legs and interfaces (to measure the relative motion between the two). A tape (green) was used to keep the electrode wiring in place. Two force-based footswitches (4) were attached underneath the heel and toes to detect the gait phase. The participants wore their own shoes and clothes.

TABLE I

EXPERIMENTAL PROTOCOL

\begin{tabular}{ll}
\hline Part & \multicolumn{1}{c}{ Activity } \\
\hline 0 & - prepare the skin and place EMG electrodes in accordance with the SENIAM guidelines [39] \\
- walk 2-3 minutes on a treadmill to warm up and determine the self-selected speed
\end{tabular}

and one while treadmill speed was gradually decreasing $(5 \rightarrow 2$ $\mathrm{km} / \mathrm{h}$ ). The choice to go with preferred [2], [24], [25], as opposed to fixed [13], [18], [45] walking speed was made to decouple the effects of an enforced walking speed (the bigger the difference between fixed and preferred speed, the more the person's gait is affected by the enforced speed).

\section{Participants}

Seven healthy young adults with no previous experience walking with the active knee joint orthosis were recruited (five males and two females, age $32 \pm 7$ years, height $1.76 \pm 0.09 \mathrm{~m}$, weight $74 \pm 14 \mathrm{~kg}$ ). All participants did, however, have prior experience walking and stair climbing wearing passive knee orthosis. The number of participants is similar to [14], [27] and falls within the commonly used sample size [11], [24], [28], [45], [46]. All participants confirmed to be free of any gaitinterfering impairments, signed an informed consent following the General Data Protection Regulation (GDPR), and provided consent to use photos and videos for publication. The ethics committee of the Vrije Universiteit Brussel University Hospital approved the study (B.U.N. 143201836843).

\section{E. Metabolic cost}

Respiratory $\mathrm{O}_{2}$ consumption and $\mathrm{CO}_{2}$ production were recorded using an appropriate face mask (Cortex, Germany) and a gas analysis system (MetaMax 3B, Cortex, Germany) worn on the chest. The data of the last minute of each 4-minute block, and the last $30 \mathrm{sec}$ of $P A$ block, are used to calculate 


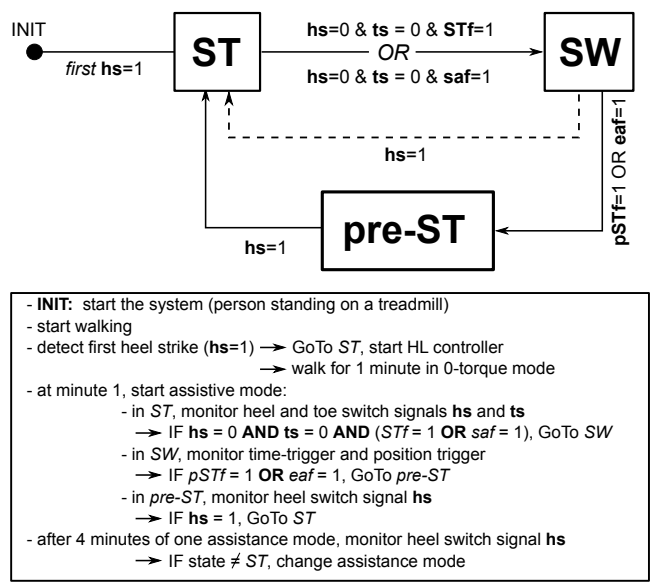

Fig. 2. Schematic representation of FSM. The FSM uses heel and toe forcebased footswitches, as well as time-based and position-based triggers to move between the states. Time-based triggers include the expected time-duration of the $S T$ phase (STf) and the pre-ST phase (pSTf), defined as \% of an average step duration (\% based on the available literature [1]). Position-based triggers include the knee joint angle indicating the start of the $S W$ phase (saf) and the upcoming heel strike (eaf). The two angles are pre-defined based on the knee biomechanics [1]. Since time-based and position-based triggers are not the main triggers, their average threshold values do not change the outcomes of the applied control framework. Two signal flows are available: the default $S T \rightarrow S W \rightarrow$ pre-ST $\rightarrow S T$ (full line) and the alternative $S T \rightarrow S W \rightarrow S T$ (dashed line).

MC using a simplified Brockway formula [47], leaving three minutes to reach metabolic steady-state [42], [48]. Net MC of walking is obtained by subtracting the standing $\mathrm{MC}$, measured at the beginning of the session, from the walking MC. The reported data, manipulated using custom-written Matlab scripts, is an average across analysed data of all participants. All values are normalized by the participant's body mass.

\section{F. Gait parameters}

Gait data were collected using a 3D motion-capture system with ten cameras (Vicon Vero 2.2, Vicon Motion Systems, United Kingdom) placed on the walls of a biomechanics lab. Each participant was fitted with a total of 30 retro-reflective markers. Of the 23 placed bilaterally in the pelvic and lowerlimbs segment, twenty were functional markers following guidelines in [49], while the extra three introduced asymmetry between the legs to allow auto labelling routine to distinguish the two (Fig. 1). The remaining seven markers were placed on the orthosis to measure its relative motion to the human.

The kinematics data were pre-processed in Vicon Nexus 2.6 and filtered using a 4th order zero-lag Butterworth filter with a $6 \mathrm{~Hz}$ cut-off frequency [1]. All analyses were carried out using custom-written scripts in Matlab 2018b (Mathworks, USA). Filtered marker coordinates were used to define right-handed local orthonormal reference frames for the pelvis and left/right thigh, shank, and foot [49]. A Cardan XYZ decomposition of the rotation matrices was used to calculate lower limb joint angles [49], corresponding to the ISB guidelines [50] of joint rotations in all three biomechanical planes.

The data were recorded throughout the entire duration of both trials. However, in post-processing and analysis, only the last minute of each 4-minute block and the last $30 \mathrm{sec}$ of the

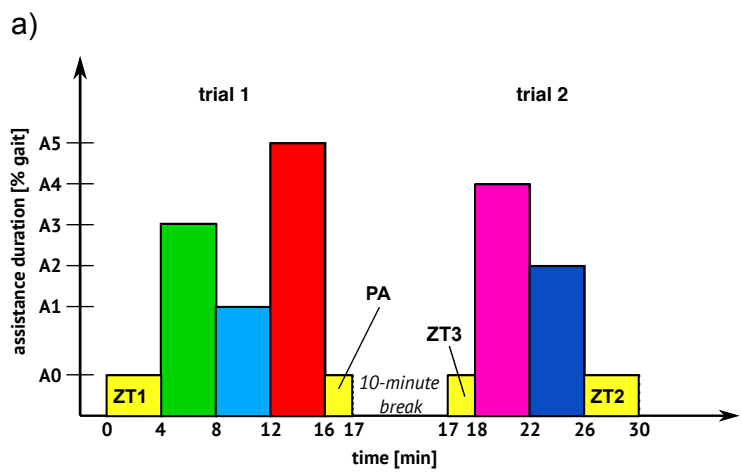

b)

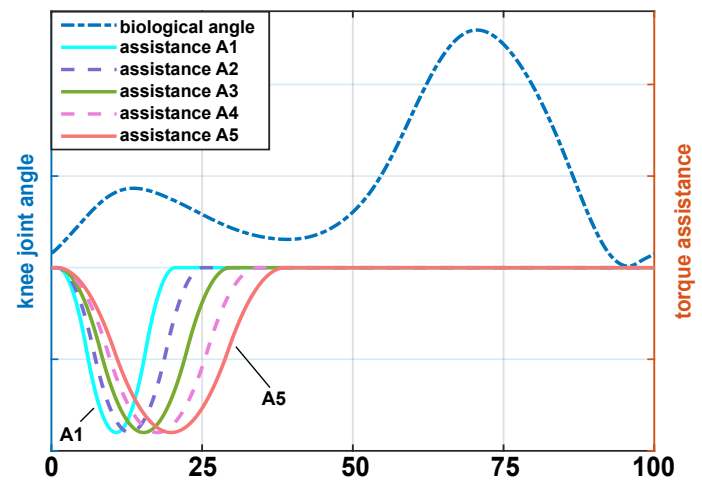

Fig. 3. Experimental protocol (example of one participant). a) The two trials are separated by a short break and start/end with a zero-torque mode (A0, in yellow). Zero-torque modes serve as follows: ZT1 as a baseline and controller set-in, ZT2 to examine learning effects and fatigue throughout the session (vs. ZT1), ZT3 as a controller set-in, and PA (Post-Assistance) to quantify the participants' immediate adaptation once assistance is turned off. $b$ ) The assistance profile is a bell-like, symmetric trajectory resembling the biological knee joint's weight-supporting torque [1] and always starting at the moment of heel strike ( $0 \%$ of a gait cycle). Assistive torque durations are fixed across all subjects, while torque amplitude changes satisfying $0.05 \mathrm{Nm} / \mathrm{kg}$.

post-assistance $(P A)$ block (minute 16-17 of trial 1, Fig. 3.a) were used. To segment gait kinematics and spatio-temporal characteristics data into gait cycles, GRFs available from the treadmill were used. Once segmented, the data were timenormalized to $0-100 \%$ using linear interpolation (heel strike to subsequent heel strike). The static calibration trial, performed while standing both with and without the orthosis, was used as a reference to define neutral joint attitudes and to correct joint angles during walking trials [49]. The reported data are an average across analysed data of all participants.

\section{G. Muscle activity}

The muscle activity of eight lower limb muscles was measured using the ME6000 biomonitor (Biomation, Canada). The surface electrodes were placed in a bipolar configuration, approx. $20 \mathrm{~mm}$ center-to-center distance, and in line with the muscle fibers [51] over the Vastus Lateralis (VL), Vastus Medialis (VM), Biceps Femoris (BF), and Gastrocnemius Medialis (GM) on each leg. Linear envelope detection was performed using a digital non-causal Finite Impulse Response (FIR) linear-phase filter (bandpass, stop-band frequencies 10 and $500 \mathrm{~Hz}$, pass-band frequencies 15 and $400 \mathrm{~Hz}$, stop-band 
attenuation $80 \mathrm{~dB}$, and $1 \mathrm{~ms}$ sampling rate), followed by a fullwave rectification and smoothening using a moving average algorithm (200 ms window). The envelopes were normalised to the peak of a one-minute average of the data recorded during normal walking $(N W)$ trial (i.e., trial without the orthosis).

The final 60 seconds of each block except $P A$ (30 seconds) were used to compute the peak (pEMG) and mean (mEMG) muscle activation in each stride. The same gait segmentation method was used as for the kinematics. To compensate for manual synchronization between the EMG and kinematic data, an assumption was made in post-processing that GM's activity goes to its minimal value at the time of a toe-off event [43], [45]. This assumption allowed performing time-based corrections to the EMG data using participant- and leg-specific patterns. The reported results are an average across analysed data of all participants. All the analyses were carried out using custom-written scripts in Matlab 2018b.

\section{H. Statistical analysis}

All statistics were calculated using an open-source software JASP v0.9.2 [52]. The analysed data include mean and peak muscle activity, MC, spatio-temporal gait characteristics (stride time, length, height, and width, and stance time), orthotic degrees of freedom (DoFs), and lower limb kinematics: hip /ankle joint ROM in the sagittal plane, and knee joint ROM in all three planes; time occurrence of the peak knee flexion and hip extension; knee joint flexion and time occurrence of the peak knee flexion during the stance phase. The analyses were carried out using paired-samples ttest and one-way repeated measures ANOVA (RMANOVA).

The relevant assumptions were inspected and corrected if needed during each test. When normality was violated, a Wilcoxon's signed-rank test or Friedman's RMANOVA were used. Confidence levels are set to $99 \%$, leading to a significance level of $\alpha=0.005$. To circumvent welldocumented flaws of using a dichotomous $p$-based significance testing [53], [54], the $p=0.01$ is set as a significance threshold, while $p<0.06$ is considered borderline significant.

Furthermore, the results are also analysed using the effect size and confidence intervals where applicable, and take biological relevance into account during the results' interpretation (e.g., a difference of 1 degree can be statistically significant but biologically insignificant if a joint has 90-degree ROM). The effect size was large if $\omega^{2}>0.14$ ( $\omega^{2}$ used due to its unbiased nature [55], [56]), and if Cohen's $|d|>0.8$ [56].

\section{RESULTS}

\section{A. Metabolic cost}

The initial RMANOVA analysis showed that some assistive modes had a statistically significant effect on changes in the net MC $(p<0.001)$ with a large effect size $\left(\omega^{2}=0.17\right)$. However, a post-hoc analysis revealed no statistically significant effects across any pair of the nine conditions (Fig. 4). This is despite the large effect size between $N W$ and all orthotic conditions and an increase in the net metabolic effort of walking during assistive conditions (min $23 \%$ in $A 3$ and $\max 27 \%$ in $A 4$ compared to $N W$ ). An increase in metabolic

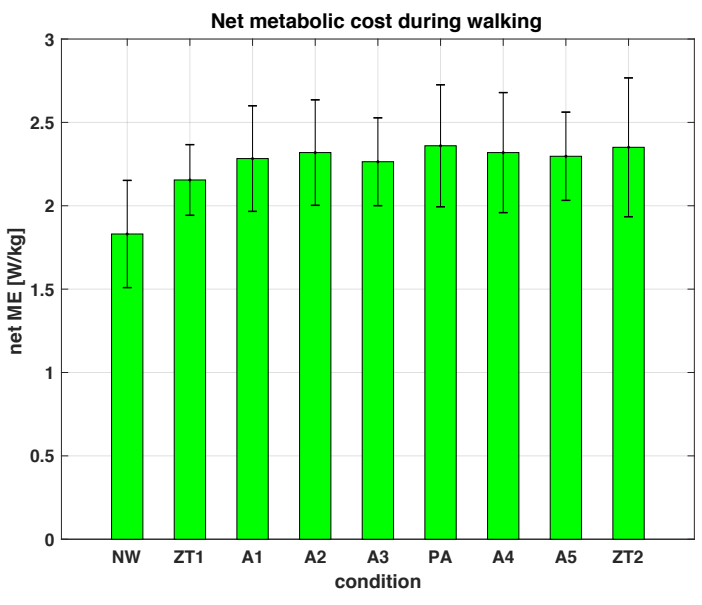

Fig. 4. Net metabolic cost during walking with the orthosis. The data is an average across all participants.

cost during zero-torque conditions $Z T 1, P A$, and $Z T 2$ compared to $N W$ is $18 \%, 29 \%$, and $28 \%$, respectively.

\section{B. Gait parameters}

Assisting the left leg had no effects on the participants' right (unassisted) leg kinematics, irrespective of the assistive condition. On the other hand, assistance affected the assisted leg's knee $\left(p<0.001, \omega^{2}=0.26\right)$ and hip $\left(\chi^{2}=23.28, p<\right.$ $0.001)$ joint's ROM in the sagittal plane, and knee flexion $\left(p<0.001, \omega^{2}=0.12\right)$ and timing of the peak knee angle $\left(\chi^{2}=15.35, p=0.02\right)$ in the stance phase (Fig. 5). All these parameters except the peak flexion timing show a difference between $N W$ and all other conditions. Differences across assistive modes are only pronounced in the timing of the peak knee stance phase angle, whose shift ranges from a minimum of $0.5 \%$ in $A 5$ to a maximum of $7 \%$ gait cycle in ZT1.

For data analysis only, we define the first three gait cycles of the $P A$ block as $C 13$ sub-block. Fig. 6 compares the hip, knee, and ankle joint gait trajectories across different blocks, including C13. Wearing the orthosis affected the hip joint mostly in the mid-gait (decreased peak extension) and ankle joint mainly in the late stance/swing phase (decreased peak plantarflexion and increased dorsiflexion). This can be seen by comparing red and green trajectories against the black one. Different can be seen in the knee joint, which was affected both in the swing (decreased peak flexion) and stance (decreased ROM and shifted peak flexion timing) phases. The assistance is able to correct time-offset but not decreased ROM in the stance phase (green vs. red trajectories).

A comparison of blue and green trajectories in the knee (Fig. 6.b) demonstrates that - from the joint kinematics point of view - participants did get affected by and rely on the provided assistance (peak flexion change of around $70 \%$ or $7^{\circ}$ compared to ZT1; see Appendix A). However, this increase washes out within about 30 seconds after switching off the assistance. This can be seen by comparing the knee joint's stance kinematics (flexion ROM and peak flexion timing) just after the assistance (blue trajectory) and during steady-state zero-torque modes (red trajectories). The fact that the latter 

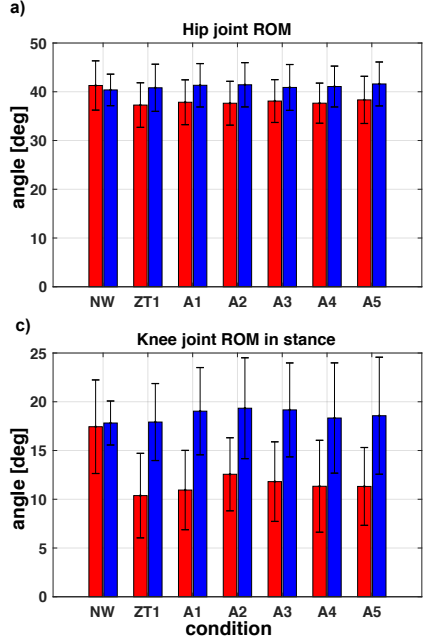

b)
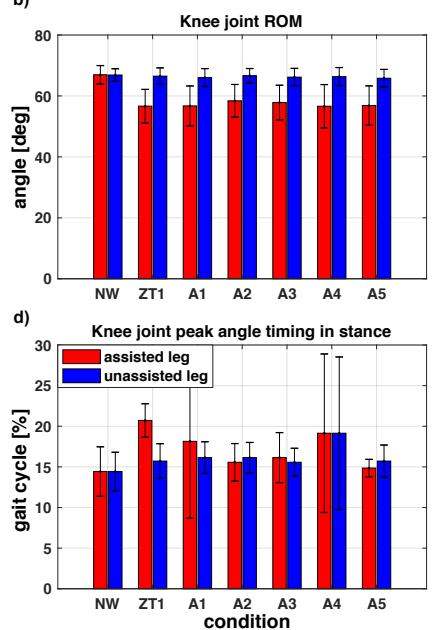

Fig. 5. Average kinematic parameters during different assistive conditions. $a-b)$ The assistive conditions affected only the knee and hip joint ROM in the sagittal plane, albeit without apparent cross-condition differences. $c$ - $d$ ) During the assisted early stance phase, differences exist across assistive conditions in both the knee joint's ROM and the timing of its peak flexion occurrence. The only parameter with significant differences across assistive conditions is the timing of the knee joint's peak flexion angle during the stance gait phase.
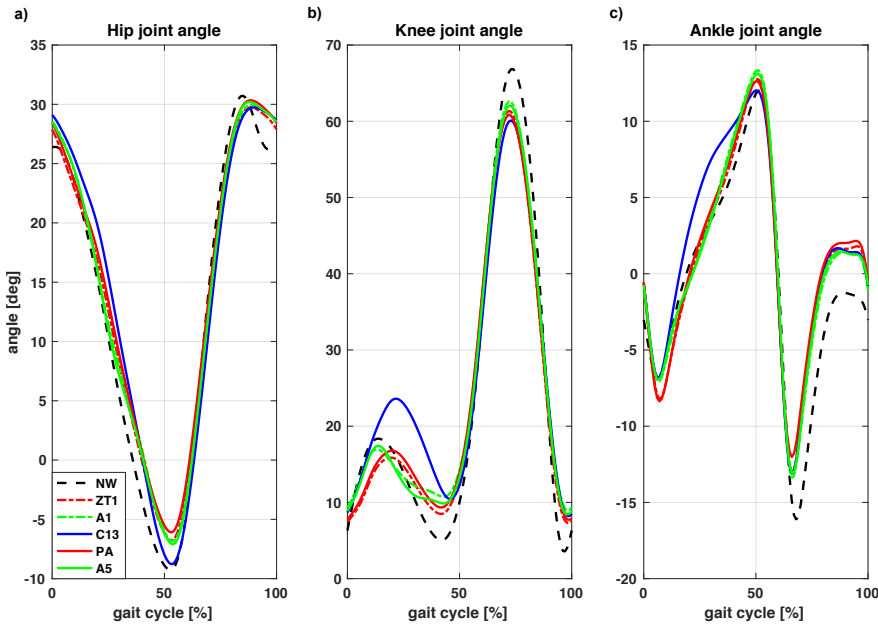

Fig. 6. Average assisted leg joint angles across different assistive conditions during walking. The conditions displayed are normal walking $N W$; zero-torque walking ZT1 (minute 0-4) and PA (minute 16-17); assisted walking $A 1$ and $A 5$; and $C 13$ (the first three gait cycles of $P A$ ). Joints are differently affected by the orthosis, both in terms of the amplitude and timing of joint trajectories. The knee joint was affected the most, particularly during the phase when assistance is provided. A decrease in the joint's extension angle in the late stance phase (35-50\% of the gait) in both zero-torque and assistive modes is a consequence of orthotic constraints.

two match $(P A \approx Z T 1)$ demonstrates convergence from a perturbation-like change in assistance captured by $C 13$ to a steady-state zero-torque $P A$ trajectory. A similar washout effect can be seen in the ankle and hip joints affected in the mid-stance and late stance gait phases, respectively, which converged within $30 \mathrm{sec}$ to their baseline trajectory ZT1.

\section{Muscle activity}

The use of the orthosis did not statistically significantly affect unassisted leg's muscle activation, despite increasing the

muscles' pEMG. The increase was dependent on the assistive condition, with a maximum of $16 \%$ for GM (ZT1), $12 \%$ for BF (Al), 27\% for VL (A3), and 12\% for VM (Al) (Fig. 7). Assistance had no statistically significant effects on the assisted leg's muscles either, but it led to assistive modedependent responses. In terms of mEMG activity, both VL and VM increased their average effort (medium effect size, $\omega^{2}=0.09$ and $\omega^{2}=0.12$, respectively), with a maximum of $20 \%$ (VL, $A 1$ and $A 2$ ) and 15\% (VM, Al). Muscle efforts of $\mathrm{BF}$ decreased in all assistive conditions, most significantly in $A 1$ and $A 2$. In terms of pEMG, assistance affected all muscles, leading to an increase of maximum/minimum $17 \% / 9 \%$ in GM (ZT1/A4), 31\%/17\% in VL (A4/ZT1), and $29 \% / 7 \%$ in VM (A1/A3). The only muscle with a decrease was BF, whose peak reduced by a maximum/minimum of $22 \% / 7 \%$ (A2/A5).

Wearing the orthosis had a similar effect throughout gait on the assisted leg's GM and BF across different assistive blocks, increasing the GM's activity during the toe-off and decreasing the BF's activity in the late swing/early stance phase (Fig. 8). Switching the assistance off had no significant effects on either of the two muscles ( $P A$ vs. C13). Different can be seen in the quadriceps muscle group. Wearing the orthosis increased both pEMG and mEMG activity of VL and VM in all assistive blocks, including zero-torque, likely due to actuator/interfaceimposed constraints. Switching off the assistance affected both their pEMG (VM) and total muscle effort (VM and VL), notably in $20-40 \%$ of the gait cycle, demonstrating, from the muscle activity point of view, that participants did rely on the assistance. However, unlike joint kinematics, it takes more than a minute for the effects of switching off the assistance to wash out, i.e., for the muscles' perturbation-compensatory activation to diminish. This is seen by the lack of a complete overlapping of $P A$ and $Z T 1$ in the muscles (Fig. 8).

\section{Discussion}

This study aimed to investigate human musculoskeletal (joint kinematics and muscle activity) and energetic (MC) adaptations and motor learning in young, healthy adults in the presence of unilateral robotic knee joint assistance during treadmill walking. In particular, the goal was to explore the importance and implications of changing a knee joint assistive torque duration. This parameter was shown to play a significant role when assisting the ankle [11], [15], and hip [16], [17] joints, but studies analysing its effects when assisting the knee joint are missing.

\section{A. Metabolic cost}

In our study, changing assistive torque duration did not lead to statistically significant differences in the net MC of walking despite some mild trends (Fig. 4). Assuming that humans are sensitive to the knee joint's assistive torque duration (similar to the, e.g., ankle [7]), at least two factors contributed to this sensitivity not being seen in our study. On the one hand, small knee joint positive mechanical work during walking [1] and weight-penalty coming from wearing an orthosis [33] led to an increase in the net MC of walking, irrespective of walking conditions. The increase is similar to the one measured in 

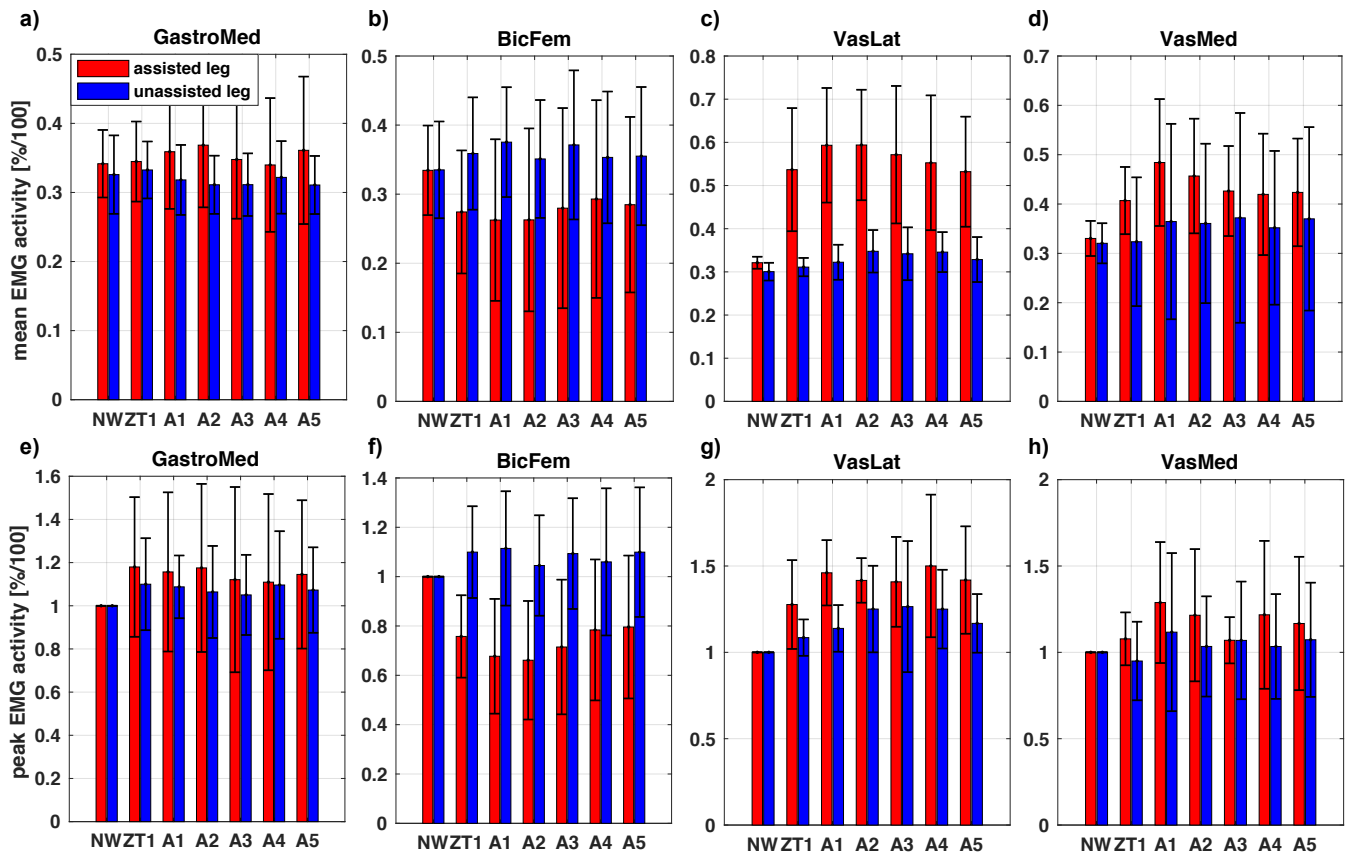

Fig. 7. Average mean and peak EMG activity. All seven participants' data are used in all muscles except VM, where one participant's data were unusable. The top row (a-d) shows mean, and the bottom row (e-h) peak EMG activity of the four muscles for all assistive conditions averaged across all participants. The assisted and unassisted leg's muscle activities are shown side-by-side for comparison. Note different y-scale. All data are normalized to peak EMG obtained during $N W$. The only muscle with a decrease in activity is the assisted leg's BF, a consequence of orthotic constraints, which slowed down the leg in the late swing phase. On the other hand, the quadriceps group muscles (VL and VM) increased their activity in all conditions involving the orthosis, albeit not to the same degree. In particular, a trend can be seen where both muscles show a decrease in mean EMG activity with an increase in assistance duration and minimum peak EMG activity during $A 3$ assistive mode.
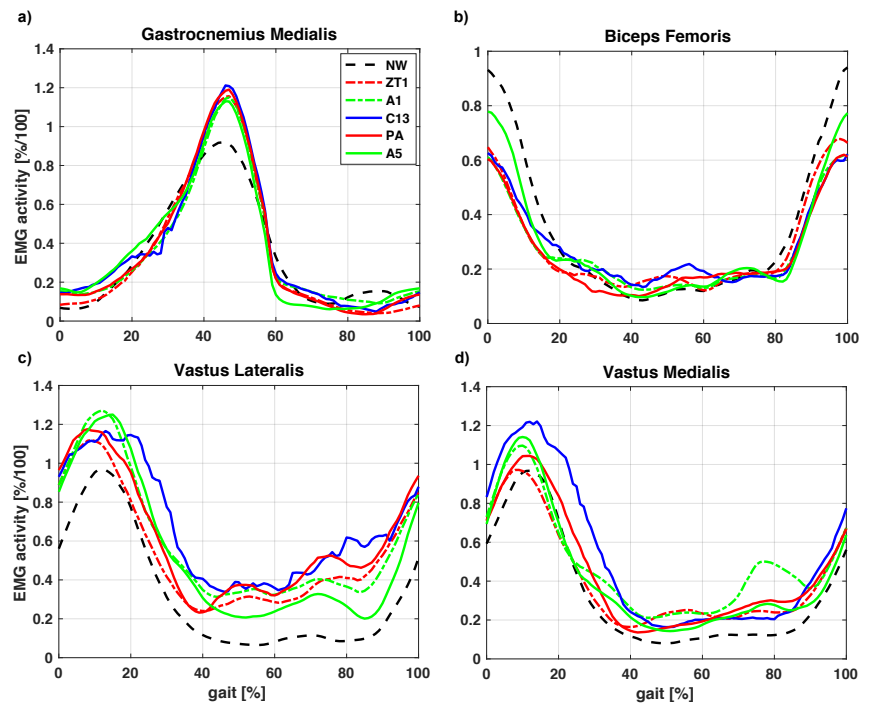

Fig. 8. Short-term post-assistance effects on muscles. GM (a) and BF (b) muscle's average gait activity is not affected much by switching the assistance off in minute 17 of trial 1 (C13 vs. PA). On the other hand, VL (c) and VM (d) are affected as their peak (VM) and total effort (VM and VL) changed right after the assistance was switched off. VL is also noticeably affected during the late stance and swing phases, when the actuator's inertia, not fully compensated by the controller, constrains participants' natural knee motion.

[24] and largely comes from the orthotic mass. Namely, we measured an increase in the net MC of walking by about $15 \%$ compared to $N W$ during preliminary experiments with a passive orthosis (not published).
On the other hand, the actuator was limited to provide a relatively low assistance $(0.05 \mathrm{Nm} / \mathrm{kg}$ vs., e.g., $0.4 \mathrm{Nm} / \mathrm{kg}$ in [22], [27]) due to the knee hyper-extension concerns during the shortest assistive torque duration. This small assistive torque was insufficient to overcome the orthotic mass effects, a dominant factor from a walking energetics point of view. In other words, low levels of assistance likely resulted in more effort from the participants trying to adapt and stabilise their gait in the presence of external 'disturbance' (dynamics) than they benefited them in assisting the most demanding part of the gait cycle [25]. Consequently, the potential importance of the knee joint's assistive torque duration on the net MC of walking could not be demonstrated in our work.

\section{B. Gait parameters}

The locality of the kinematics effects in the presence of external assistance, independent of the assistive condition, confirmed the first hypothesis. This is in line with the kinematic effects of wearing a passive unilateral knee orthosis [2]. The orthosis did not cause fatigue either, nor did it introduce short-term (within-session, ZT1 vs. ZT2) motor learning effects in any analysed DoF except the assisted leg's hip joint's ROM ( $p<0.001, d=-1.65)$. However, as Fig. 5 shows, it did have an assistive mode-dependent effects on gait parameters of the assisted leg. These include decreasing the leg's stride height by a maximum of $8 \%(26 \rightarrow 24 \mathrm{~cm})$, hip joint's ROM by a maximum of $10 \%\left(41.3^{\circ} \rightarrow 37.3^{\circ}\right)$, and the knee joint's ROM by a maximum of $18.5 \%\left(67^{\circ} \rightarrow 56.5^{\circ}\right)$. 
Comparing these results to preliminary tests with passive orthosis suggests that the changes in the hip joint's ROM come from the orthotic mass/articulation, rather than from the assistance (bilateral orthotic assistance at the knee joint level also affects leg kinematics only locally [25]). Different can be seen in the knee joint (Fig. 6.b), whose ROM decreased while providing assistance for an additional $8^{\circ}(15 \%)$ on top of $2^{\circ}$ measured during the preliminary tests. This comes from the lack of full orthotic transparency, i.e., the inability to fully compensate for orthotic dynamics. This is a common problem with WRs [57], particularly noticeable during fast motions such as the knee joint's swing phase (the actuator's impedance and backdriving torque can be found in [35]).

On the other hand, a decrease in the knee joint's ROM in the early stance phase (Fig. 5.c and Fig. 6.b) can mainly be attributed to the actuator's so-called dead-zone, a workspace around zero torque where the actuator's torque output is not fully accurate [34], [35]. During the early stance phase, the knee joint's flexion closely follows that of $N W$ as long as the assistance is provided to the joint (green vs. dotted black trajectory, Fig. 6.b). When the assistance is switched off, the two trajectories diverge, leading to a decrease up to $60 \%\left(7^{\circ}\right)$ in the knee joint's late stance extension. A similar result can be seen during zero-torque blocks ZT1 and $P A$ (red vs. dotted black trajectory, Fig. 6.b), where the reduction in the knee joint's ROM is accompanied by a shift (delay) in time (up to $7 \%$ of the gait cycle, Fig. 5.d).

The only gait parameter with a significant difference across assistive conditions is the knee joint's peak flexion timing during the stance phase (Fig. 5.d). The timing was invariant to assistance duration in the case of assistive conditions $A 2, A 3$, and $A 5$. The change in timing during assistive condition $A 1$ is due to an impulse-like assistive profile, which also had the highest negative impact on muscle activity (and was a limiting factor on the peak assistance). On the other hand, a significant change in timing during the assistive condition $A 4$, seen in both assisted and unassisted leg, could likely be explained by the resonance in the biological and external torque timings. The peak torque timing of the assistive condition $A 4$ comes at about $15 \%$ of the gait cycle, which coincides with the biological peak torque timing of a typical gait [58].

This suggests that humans tend to keep the timing of their key stance event invariant to external disturbance/assistance, a result in line with earlier works on the human-robot interaction at the knee level. Like our result, Shirota [2] showed that wearing (passive) knee orthosis reduces the peak knee flexion in the swing phase but has no effect on the peak flexion timing. On the other hand, Shamaei [25] demonstrated that humans regulate their knee joint quasi-stiffness to keep the overall human-robot quasi-stiffness invariant. Interestingly, Shamaei et al. showed invariance to hold across a wide but not the full range of knee joint quasi-stiffness. This is in line with our results in that both works suggest limitations in the knee joint's response to adjust to the external disturbance.

\section{Muscle activity}

Wearing the orthosis unilaterally increased the unassisted leg's VL and VM's muscle activity by $25 \%$ and $10 \%$, respectively. The biggest changes were observed during the shortest assistive conditions ( $A 1$ and $A 2)$ and irrespective of the elapsed session time, as demonstrated by the lack of learning effects or fatigue (ZT1 vs. ZT2). These compensatory muscle activities suggest a bigger contribution of the unperturbed leg to gait stabilisation in the presence of unilateral impulselike perturbation. This inter-limb communication on a muscle activity level that seems to cater to gait stability across different walking conditions has many implications. One notable is the ability to induce an impaired leg's desired behaviour by deliberately perturbing a healthy one [59].

The assisted leg's muscles show more complex responses to assistive conditions. In general, wearing the orthosis increased muscle activity. This increase is assistance-dependent and comes from two sources. On the one hand, the orthosis was not fully transparent, which made the participants back-drive the motor, increasing the quadriceps activity (e.g., VL's activity in the swing phase, Fig. 8). On the other hand, low assistance amplitude, limited by the participants' comfort, was not high enough to overcome orthotic mass and articulation's inevitable negative effects. The one muscle that benefited from wearing the orthosis was BF, whose activity was decreased in the late swing/early stance phases. The benefits of the assistance and its varying duration were nevertheless present since all participants confirmed they felt assisted but had different preferences in terms of assistance duration.

\section{Instantaneous (washout) motor learning effects}

The actual effects of assistance were visually observable during the experiments. When support was switched off in minute 17 of trial 1 (Fig. 3.a), which participants were warned about ten seconds before and at the moment of the change, the knee joint's early stance flexion ROM increased by about $70 \%$ (C13 vs. PA, Fig. 6.b). A much higher knee flexion amplitude during $C 13$ sub-block compared to other conditions suggests that the participants were affected by the assistance. It also suggests their knee joint did, at least to some extent, adjust to the support. At the same time, a good match between zerotorque mode trajectories $Z T 1$ and $P A$ suggests that it takes about 30 seconds for the knee joint kinematics (in the sagittal plane) to recover from a sudden change in assistive mode. This is because $C 13$ trajectory converges to an average $P A$ trajectory within the first 30 seconds after the assistance is switched off. Such a fast convergence validates the use of 4-minute blocks with the first three minutes used as an adaptation period.

Similar to the joint kinematics, a sudden loss of assistance in minute 17 of trial 1 (Fig. 3.a) also affected the assisted leg's muscle activity (Fig. 8), resulting in visible changes in both VM and VL muscles. Compared to ZT1, removing assistance increased VM's mEMG by $30 \%$ and VL's mEMG by $19 \%$ in the early stance gait phase, in addition to a time-shift in VL's peak activity (7\% of the gait cycle to the right) and an increase in VM's amplitude (25\%). However, muscles needed more time to stabilise after the change in activity than joints did. A comparison of muscle activity in ZTI and PA zerotorque modes with $C 13$ sub-block (Fig. 8) shows that muscles need more than a minute, albeit seemingly not much more, to 
adjust to a new condition (even when controlling for fatigue and learning effects).

In other words, alterations to the leg's main extensor muscles caused by external assistance are not necessarily seen in the joint's kinematics. Between the two, the joint shows higher levels of damping and resilience to external perturbations than muscles. This is because the joint motion is a consequence of active muscle tissue and the workings of the passive elements, such as ligaments and tendons, which act as a buffer in absorbing external perturbations.

\section{E. Study limitations}

Due to data collection equipment's incompatibility, synchronisation of the muscle activity and joint kinematics data had to be done manually in post-processing. Despite doing the synchronisation following the literature, this could have affected the measured joint kinematics and muscle activity's exact relative timing. However, small potential offsets in the two parameters' temporal profile do not change the nature of human body adaptations to the external knee joint assistance captured during the experiments or alter the conclusions presented in this paper.

A sudden buckle at the knee joint, followed by a recovery phase, demonstrates that participants did get affected by the assistance despite being informed about the upcoming change twice. Arguably, these warnings could have affected results and minimise the actual changes in the participants' gait but were used nevertheless for safety reasons. The results could have been affected by the protocol design as well, but it is important to note that no agreement exists in the field on the right methodology and/or time-duration of the protocol. On the other hand, the recovery phase captured in this work demonstrates that four minutes in each condition is sufficient in terms of joint kinematics and muscle activity to get stable short-term human adaptation to external assistance.

\section{Conclusions}

This study presented insights on the effects that unilateral robotic assistance provided to the human knee joint has on healthy young adults' metabolic cost, muscle effort, and gait parameters. By exploring the importance and implications of changing assistive torque duration, this study was a step towards filling the gap in our understanding of the human body's adaptation mechanisms associated with the use of WRs, which remain an active research area.

The results show for the first time that the human body adapts its lower limb joint kinematics and muscle activity differently to external knee assistance. Kinematically, the effects of unilateral knee joint assistance remain mainly local to the assisted joint, while muscles spanning the knee joint get affected on both legs. On the unassisted leg, this seems to be a balance-related compensatory activation.

Short-term post-assistance effects in terms of joint kinematics and muscle activity demonstrate that participants did get affected by the support they were provided. However, joint kinematics are quicker than muscle activity in recovering from the removal of the external assistance due to passive tissues surrounding the joint, demonstrating different washout effects on the joint and muscular levels.

Finally, changing assistive torque duration statistically significantly affected only the timing of the knee joint's peak flexion angle during the stance phase. The occurrence of the changes suggests that limitations exist in the knee joint's ability to adjust to the external disturbance.

\section{APPENDIX A}

A video (.mp4) is added to this paper, demonstrating the short-term post-assistance effects right after the assistance is switched off.

\section{ACKNOWLEDGMENT}

The authors would like to thank Prof. Dr. Eva Swinnen, Dr. Nina Lefeber, and Drs. Emma De Keersmaecker of the Faculty of Physical Education and Physiotherapy of the Vrije Universiteit Brussels for their contribution in preparing the experimental study and assistance in using EMG and gas measuring apparatus. The authors would also like to thank the subjects participating in the study for their cooperation and dedication.

\section{REFERENCES}

[1] D. Winter, Biomechanics and Motor Control of Human Movement, Fourth Edition. Wiley, sep 2009.

[2] C. Shirota, M. R. Tucker, O. Lambercy, and R. Gassert, "Kinematic effects of inertia and friction added by a robotic knee exoskeleton after prolonged walking," in Int. Conf. Rehabil. Robot., 2017, pp. 430-434.

[3] R. W. Jackson and S. H. Collins, "Heuristic-Based Ankle Exoskeleton Control for Co-Adaptive Assistance of Human Locomotion," IEEE Transactions on Neural Systems and Rehabilitation Engineering, vol. 27, no. 10, pp. 2059-2069, 2019.

[4] S. J. Ali, A. N. Ansari, A. Rahman, S. Imtiyaz, and B. Rashid, "Poststroke hemiplegic gait: A review," Pharma Innov., vol. 3, no. 8, pp. 36-41, 2014.

[5] Y. P. Ivanenko, R. E. Poppele, and F. Lacquaniti, "Five basic muscle activation patterns account for muscle activity during human locomotion." The Journal of physiology, vol. 556, no. Pt 1, pp. 267282, apr 2004.

[6] A. J. Young and D. P. Ferris, "State-of-the-art and Future Directions for Lower Limb Robotic Exoskeleton," IEEE Trans. Neural Syst. Rehabil. Eng., vol. 25, no. 2, pp. 171-182, 2016.

[7] M. Moltedo, T. Bacek, T. Verstraten, C. Rodriguez-Guerrero, B. Vanderborght, and D. Lefeber, "Powered Ankle-foot Orthoses : The Effects of the Assistance on Healthy and Impaired Users While Walking," J. Neuroeng. Rehabil., vol. 15, no. 86, 2018.

[8] J. Kim, G. Lee, R. Heimgartner, D. Arumukhom Revi, N. Karavas, D. Nathanson, I. Galiana, A. Eckert-Erdheim, P. Murphy, D. Perry, N. Menard, D. K. Choe, P. Malcolm, and C. J. Walsh, "Reducing the metabolic rate of walking and running with a versatile, portable exosuit," Science, vol. 365, no. 6454, pp. 668 - 672, aug 2019.

[9] G. S. Sawicki, O. N. Beck, I. Kang, and A. J. Young, "The exoskeleton expansion: improving walking and running economy," Journal of NeuroEngineering and Rehabilitation, vol. 17, no. 1, p. 25, 2020.

[10] M. Wehner, B. Quinlivan, P. M. Aubin, E. Martinez-Villalpando, M. Baumann, L. Stirling, K. Holt, R. Wood, and C. Walsh, "A lightweight soft exosuit for gait assistance," in 2013 IEEE International Conference on Robotics and Automation, 2013, pp. 3362-3369.

[11] P. Malcolm, W. Derave, S. Galle, and D. De Clercq, "A Simple Exoskeleton That Assists Plantarflexion Can Reduce the Metabolic Cost of Human Walking," PLoS One, vol. 8, no. 2, pp. 1-7, 2013.

[12] L. M. Mooney, E. J. Rouse, and H. M. Herr, "Autonomous exoskeleton reduces metabolic cost of human walking during load carriage," $J$. Neuroeng. Rehabil., vol. 11, no. 1, p. 80, 2014. 
[13] J. R. Koller, D. H. Gates, D. P. Ferris, and C. D. Remy, "Confidence in the curve: Establishing instantaneous cost mapping techniques using bilateral ankle exoskeletons," Journal of Applied Physiology, vol. 122(2), pp. 242-252, 2016.

[14] B. T. Quinlivan, S. Lee, P. Malcolm, D. M. Rossi, M. Grimmer, C. Siviy, N. Karavas, D. Wagner, A. Asbeck, I. Galiana, and C. J. Walsh, "Assistance magnitude versus metabolic cost reductions for a tethered multiarticular soft exosuit," Sci. Robot., vol. 2, no. 2, jan 2017.

[15] J. Zhang, P. Fiers, K. A. Witte, R. W. Jackson, K. L. Poggensee, C. G. Atkeson, and S. H. Collins, "Human-in-the-loop optimization of exoskeleton assistance during walking," Science, vol. 356, no. 6344, pp. 1280-1284, 2017.

[16] Y. Ding, F. Panizzolo, C. Siviy, P. Malcolm, I. Galiana, K. Holt, and C. Walsh, "Effect of timing of hip extension assistance during loaded walking with a soft exosuit," Journal of NeuroEngineering and Rehabilitation, vol. 13, oct 2016

[17] J. Lee, K. Seo, B. Lim, J. Jang, K. Kim, and H. Choi, "Effects of assistance timing on metabolic cost, assistance power, and gait parameters for a hip-type exoskeleton," in 2017 International Conference on Rehabilitation Robotics (ICORR), 2017, pp. 498-504.

[18] A. T. Asbeck, S. M. M. De Rossi, K. G. Holt, and C. J. Walsh, "A biologically inspired soft exosuit for walking assistance," Int. J. Rob. Res., vol. 34, no. 6, pp. 744-762, mar 2015.

[19] Y. Ding, I. Galiana, A. Asbeck, S. M. M. De Rossi, J. Bae, T. Santos, V. Araújo, S. Lee, K. Holt, and C. Walsh, "Biomechanical and Physiological Evaluation of Multi-Joint Assistance With Soft Exosuits," IEEE Transactions on Neural Systems and Rehabilitation Engineering, vol. 99, p. 1, jan 2016.

[20] F. A. Panizzolo, I. Galiana, A. T. Asbeck, C. Siviy, K. Schmidt, K. G. Holt, and C. J. Walsh, "A biologically-inspired multi-joint soft exosuit that can reduce the energy cost of loaded walking," J. Neuroeng. Rehabil., vol. 13, no. 1, p. 43, 2016.

[21] C. J. Walsh, K. Endo, and H. Herr, "A quasi-passive leg exoskeleton for load-carrying augmentation," Int. J. Humanoid Robot., vol. 04, no. 03, pp. 487-506, sep 2007.

[22] Z. Zhou, Y. Liao, C. Wang, and Q. Wang, "Preliminary evaluation of gait assistance during treadmill walking with a light-weight bionic knee exoskeleton," in 2016 IEEE International Conference on Robotics and Biomimetics (ROBIO), 2016, pp. 1173-1178.

[23] J. Jang, B. Lim, and Y. Shim, "An assistance approach for a powered knee exoskeleton during level walking and the effects on metabolic cost," in 201941 st Annual International Conference of the IEEE Engineering in Medicine and Biology Society (EMBC), 2019, pp. 6216-6219.

[24] M. K. MacLean and D. P. Ferris, "Energetics of Walking With a Robotic Knee Exoskeleton," Journal of Applied Biomechanics, vol. 35, no. 5, pp. 320-326, 2019.

[25] K. Shamaei, M. Cenciarini, A. A. Adams, K. N. Gregorczyk, J. M. Schiffman, and A. M. Dollar, "Biomechanical Effects of Stiffness in Parallel With the Knee Joint During Walking," IEEE Trans. Biomed. Eng., vol. 62, no. 10, pp. 2389-2401, 2015.

[26] A. Gams, T. Petrič, T. Debevec, and J. Babič, "Effects of Robotic Knee Exoskeleton on Human Energy Expenditure," IEEE Trans. Biomed. Eng., vol. 60, no. 6, pp. 1636-1644, 2013.

[27] Z. F. Lerner, D. L. Damiano, and T. C. Bulea, "The Effects of Exoskeleton Assisted Knee Extension on Lower-Extremity Gait Kinematics, Kinetics, and Muscle Activity in Children with Cerebral Palsy," Scientific Reports, vol. 7, no. 1, pp. 1-12, 2017.

[28] F. Lerner, D. Damiano, and T. Bulea, "Relationship between assistive torque and knee biomechanics during exoskeleton walking in individuals with crouch gait," in 2017 International Conference on Rehabilitation Robotics (ICORR), 2017, pp. 491-497.

[29] G. Elliott, G. S. Sawicki, A. Marecki, and H. Herr, "The biomechanics and energetics of human running using an elastic knee exoskeleton," in IEEE Int. Conf. Rehabil. Robot., 2013.

[30] M. E. Roebroeck, C. A. M. Doorenbosch, J. Harlaar, R. Jacobs, and G. J. Lankhorst, "Biomechanics and muscular activity during sit-to-stand transfer," Clinical Biomechanics, vol. 9, no. 4, pp. 235-244, jul 1994.

[31] K. Shamaei, P. C. Napolitano, and A. M. Dollar, "Design and Functional Evaluation of a Quasi-Passive Compliant Stance Control Knee-AnkleFoot Orthosis," IEEE Trans. Neural Syst. Rehabil. Eng., vol. 22, no. 2, pp. $258-268,2014$

[32] D. Zanotto, Y. Akiyama, P. Stegall, and S. K. Agrawal, "Knee Joint Misalignment in Exoskeletons for the Lower Extremities: Effects on User's Gait," IEEE Trans. Robot., vol. 31, no. 4, pp. 978-987, 2015.

[33] R. C. Browning, J. R. Modica, R. Kram, and G. A., "The effects of adding mass to the legs on the energetics and biomechanics of walking," Med Sci Sport. Exerc., vol. 39, no. 3, pp. 515-525, 2007.
[34] T. Bacek, M. Moltedo, C. Rodriguez-Guerrero, J. Geeroms, B. Vanderborght, and D. Lefeber, "Design and evaluation of a torquecontrollable knee joint actuator with adjustable series compliance and parallel elasticity," Mech. Mach. Theory, vol. 130, pp. 71-85, 2018.

[35] T. Baček, M. Moltedo, J. Geeroms, B. Vanderborght, C. RodriguezGuerrero, and D. Lefeber, "Varying mechanical compliance benefits energy efficiency of a knee joint actuator," Mechatronics, vol. 66, 2020.

[36] K. Langlois, M. Moltedo, T. Bacek, C. Rodriguez Guerrero, B. Vanderborght, and D. Lefeber, "Design and Development of Customized Physical Interfaces to Reduce Relative Motion between the User and a Powered Ankle Foot Exoskeleton," in IEEE Int. Conf. Biomed. Robot. Biomechatronics, Accept., 2018.

[37] A. D. Kuo, J. M. Donelan, and A. Ruina, "Energetic Consequences of Walking Like an Inverted Pendulum: Step-to-Step Transitions," Exerc. Sport Sci. Rev., vol. 33, no. 2, 2005.

[38] D. A. Winter, Knee Flexion During Stance as a Determinant of Inefficient Walking, apr 1983, vol. 63.

[39] "Seniam," 2019. [Online]. Available: http://www.seniam.org

[40] A. Leardini, Z. Sawacha, G. Paolini, S. Ingrosso, R. Nativo, and M. G. Benedetti, A new anatomical based protocol for gait analysis in children, nov 2007, vol. 26

[41] C. A. Fukuchi, R. K. Fukuchi, and M. Duarte, "A public dataset of overground and treadmill walking kinematics and kinetics in healthy individuals," PeerJ, vol. 6, 2018.

[42] P. Malcolm, D. M. Rossi, C. Siviy, S. Lee, B. T. Quinlivan, M. Grimmer, and C. J. Walsh, "Continuous sweep versus discrete step protocols for studying effects of wearable robot assistance magnitude," Journal of NeuroEngineering and Rehabilitation, vol. 14, no. 1, p. 72, 2017.

[43] S. Galle, P. Malcolm, S. H. Collins, and D. De Clercq, "Reducing the metabolic cost of walking with an ankle exoskeleton: interaction between actuation timing and power," J. Neuroeng. Rehabil., vol. 14, no. 1, 2017.

[44] J. C. Selinger and J. M. Donelan, "Estimating instantaneous energetic cost during non-steady-state gait," Journal of Applied Physiology, vol. 117, no. 11, pp. 1406-1415, sep 2014.

[45] P. Malcolm, S. Galle, W. Derave, and D. De Clercq, "Bi-articular KneeAnkle-Foot Exoskeleton Produces Higher Metabolic Cost Reduction than Weight-Matched Mono-articular Exoskeleton,” p. 69, 2018.

[46] S. H. Collins, M. B. Wiggin, and G. S. Sawicki, "Reducing the energy cost of human walking using an unpowered exoskeleton," Nature, 2015

[47] J. Brockway, Derivation of formulae used to calculate energy expenditure in man, dec 1987, vol. 41.

[48] W. Felt, J. C. Selinger, J. M. Donelan, and C. D. Remy, “"Body-In-TheLoop": Optimizing Device Parameters Using Measures of Instantaneous Energetic Cost," PLOS ONE, vol. 10, no. 8, aug 2015.

[49] D. Robertson, G. E Caldwell, J. Hamill, G. Kamen, and S. N Whittlesey, Research Methods in Biomechanics: Second edition (eBook), nov 2013.

[50] G. Wu, S. Siegler, P. Allard, C. Kirtley, A. Leardini, D. Rosenbaum, M. Whittle, D. D. D'Lima, L. Cristofolini, H. Witte, O. Schmid, and I. Stokes, "ISB recommendation on definitions of joint coordinate system of various joints for the reporting of human joint motion-part I: ankle, hip, and spine," Journal of Biomechanics, vol. 35, no. 4, pp. 543-548, 2002.

[51] P. Konrad, The ABC of EMG: a practical introduction to kinesiological electromyography. Noraxon USA, Inc, 2006.

[52] JASPTeam, "JASP (Version 0.9.2)[Computer software]," 2019. [Online]. Available: https://jasp-stats.org/

[53] J. Cohen, The Earth is Round ( $p<.05$ ), jan 1994, vol. 49.

[54] R. L. Wasserstein and N. A. Lazar, "The ASA's Statement on p-Values: Context, Process, and Purpose," The American Statistician, vol. 70(2), pp. 129-133, 2016.

[55] T. R. Levine and C. R. Hullett, "Eta Squared, Partial Eta Squared, and Misreporting of Effect Size in Communication Research," Human Communication Research, vol. 28, no. 4, pp. 612-625, oct 2002.

[56] M. Gross-Sampson, "Statistical analysis in JASP: A guide for students," 2018.

[57] J. Vantilt, K. Tanghe, M. Afschrift, A. K. B. D. Bruijnes, K. Junius, J. Geeroms, E. Aertbeliën, F. De Groote, D. Lefeber, I. Jonkers, and J. De Schutter, "Model-based control for exoskeletons with series elastic actuators evaluated on sit-to-stand movements," Journal of NeuroEngineering and Rehabilitation, vol. 16, no. 1, p. 65, 2019.

[58] J. Perry and J. Davids, Gait analysis: normal and pathological function. SLACK Incorporated, 1992.

[59] J. Skidmore and P. Artemiadis, "Unilateral changes in walking surface compliance evoke dorsiflexion in paretic leg of impaired walkers." Journal of rehabilitation and assistive technologies engineering, vol. 4, 2017. 


\section{University Library}

\section{- M M N E R VA A gateway to Melbourne's research publications}

Minerva Access is the Institutional Repository of The University of Melbourne

\section{Author/s:}

Bacek, T;Moltedo, M;Serrien, B;Langlois, K;Vanderborght, B;Lefeber, D;Rodriguez-Guerrero, C

Title:

Human Musculoskeletal and Energetic Adaptations to Unilateral Robotic Knee Gait Assistance

Date:

2022-03-01

Citation:

Bacek, T., Moltedo, M., Serrien, B., Langlois, K., Vanderborght, B., Lefeber, D. \& RodriguezGuerrero, C. (2022). Human Musculoskeletal and Energetic Adaptations to Unilateral Robotic Knee Gait Assistance. IEEE TRANSACTIONS ON BIOMEDICAL ENGINEERING, 69 (3), pp.1141-1150. https://doi.org/10.1109/TBME.2021.3114737.

Persistent Link:

http://hdl.handle.net/11343/285322 